\title{
Voice Message Silence Compression
}

\author{
H.N.Yadav \\ V.B.S Purvanchal University, Jaunpur \\ *Corresponding Author: hnyadav_vbspu@yahoo.com
}

Copyright (C) 2014 Horizon Research Publishing All rights reserved.

\begin{abstract}
Silence compression algorithm for voice message compresses sound files. In This paper software shows greater efficiency for the sound files having number of silence runs. It can be used in telecommunication for sending voice messages in compressed form the advantage of context adaptive voice message Silence compression is that it frequently smooths out the data, which makes it even more suitable [4]. In This paper we get an extra unexpected benefit from lossy compression, above and beyond the compression itself. Silence compression software has successfully been tested for 25 wav files sampled at $8000 \mathrm{hz}, 16$ bits .
\end{abstract}

Keywords Context Adaptive Lossless Image Compression (CALIC), Lossless Compression

\section{Voice Compression}

Manipulation of sound by computers is possible since the birth of digital computers, but only in the last five years or so has inexpensive hardware brought this to the average user's desktop. Now the ability to play digitized sound is expected to be an integral part of the "multimedia revolution." The use of multimedia focuses the issue of data compression for users. Computer graphics in particular quickly take up all available disk space. Digitized audio is far less voracious in its storage requirements, but even so it can quickly swallow up all free space on the average user's hard disk. Fortunately for computer users, the world of telephony has used digitized audio since the 1960s, and extensive research has been done on effective methods of encoding and compressing audio data. The world's telecommunications companies were intensely aware of the cost of transmission bandwidth and made efforts to reduce expenses in this area [1].

\section{Voice Compression Techniques}

\subsection{Lossless Compression of Sound}

In this technique sound is compressed without any lossy in data,the compressed data can be decompressed to its actual form. Lossy compression of sound The very word "lossy" implies that when using this type of compression we are going to give up a certain amount of precision [4]. This would certainly not be acceptable when compressing the data or text files we use on our computers.

\subsection{In Lossy Compression}

In lossy compression we accept a certain loss in fidelity. The signal we get after going through a compression/expansion cycle will not be identical to the original, but we can adjust the parameters to get better or worse fidelity, and likewise better or worse compression. Lossy compression is not necessarily an end to itself. We frequently use lossy compression in a two-phase process: a lossy stage followed by a lossless stage [4]. One advantage about lossy compression is that it frequently smooths out the data, which makes it even more suitable for lossless compression. So we get an extra unexpected benefit from lossy compression, above and beyond the compression itself.

\subsection{Context Adaptive Voice Message Silence Compression}

Context adaptive algorithm of Silence compression on sound files is the equivalent of run-length encoding on normal data files. In this case, however, the runs we encode are sequences of relative silence in a sound file. This is a lossy technique because we replace the sequences of relative silence with absolute silence [3].

\section{Digital Audio Concepts}

For modern computers to manipulate sound, they first have to convert it to a digital format. The sound samples can then be processed, transmitted, and converted back to analog format, where they can finally be received by the human ear.

Digitization of sound began in earnest in the early 1960s. Virtually all new switching equipment installed by telephone companies today is digital. But analog switching is still found in older installations and in the smaller PBX and key systems installed in businesses. Of course, the final subscriber loop between the telephone company and the end 
user is still persistently analog. Digital audio is now coming of age in the highly visible consumer electronics arena as well. The digital compact disk has nearly completed its displacement of analog LP records [5]. It remains to be seen whether digital audio tape will do the same thing to analog cassette tape, but it seems likely that someday most recorded music will be distributed in digital format.

The $\mathrm{X}$ axis in this diagram represents time. The $\mathrm{Y}$ axis represents a voltage measured at an input device, typically a microphone. The microphone attempts to faithfully reproduce changes in air pressure caused by sound waves traveling through it. Some human ears can hear sounds at frequencies as high as $20,000 \mathrm{~Hz}$ and nearly as low as DC. The dynamic range of our hearing is so wide that we have to employ a logarithmic scale of measurement, the decibel, to reasonably accommodate it. This presents a unique set of requirements for digitization[9].

A waveform like the shown in Figure 1 is typical of audio sample. It isn't a nice, clean sine wave that has a regular period and can be described as a simple mathematical function. Instead, it is a combination of various frequencies at different amplitudes and phases. When combined, we see something that looks fairly irregular and not easy to characterize.

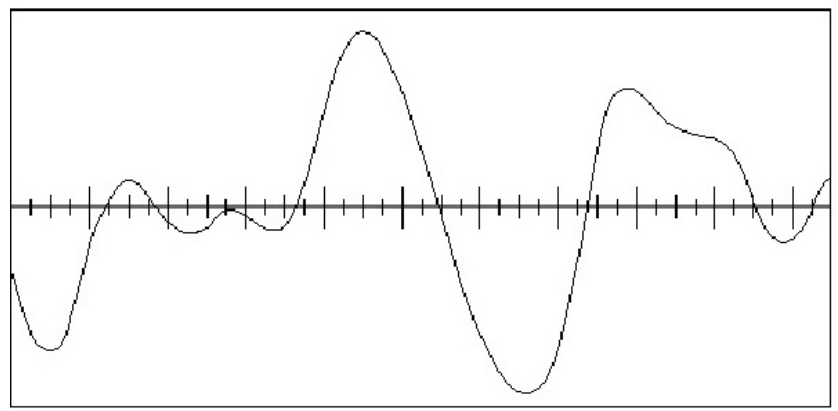

Figure 1 A typical audio waveform as it might be displayed on an oscilloscope.

This particular "snapshot" shows about 5 milliseconds (ms) of output. Notice that the largest recognizable components of the waveform appear to have a period of roughly two milliseconds. This corresponds to a frequency of about $500 \mathrm{~Hz}$, a fairly characteristic frequency found in speech or music.

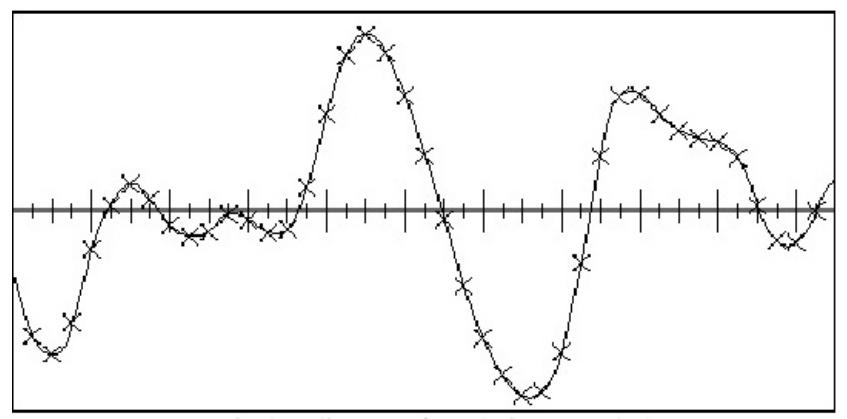

Figure 2 A typical audio waveform being sampled at $8 \mathrm{KHz}$.

The first step in working with digital audio is "sampling."
Sampling consists of taking measurements of the input signal at regular times, converting them to an appropriate scale, and storing them. Figure 2 shows the same waveform sampled at an $8 \mathrm{KHz}$ rate. This means that 8,000 times per second a measurement is taken of the voltage level of the input signal. The measurement points are marked with an " $x$ " on the waveform[11].

In most computer systems, this first step of digitization is done with an analog-to-digital converter (ADC). The ADC takes a given voltage and scales it to an appropriate digital measurement. An eight-bit ADC, for example, might have a "full scale" input voltage of 500 millivolts (mv) - it would output an eight-bit value of 255 if the input voltage were $500 \mathrm{mv}$ and zero if the input voltage were zero. A voltage between these values would be scaled to fit in the linear range of zero to 255. Since audio signals are AC in natured, the ranges are usually adjusted so that a zero voltage signal falls in the middle of the range. For the previous example, the range would be adjusted to between $-250 \mathrm{mv}$ and $+250 \mathrm{mv}$. Outputs from the eight-bit ADC would range from -128 to +127 . The stored sample points then represent a series of voltages that were measured at the input of the ADC. Figure 3 shows the representation of those voltages overlaid with the input $\mathrm{AC}$ sigma[12]1. Note that since the sample points in this case are occurring many times more frequently than the period of the waveform, the digital samples themselves trace the analog signal very accurately.

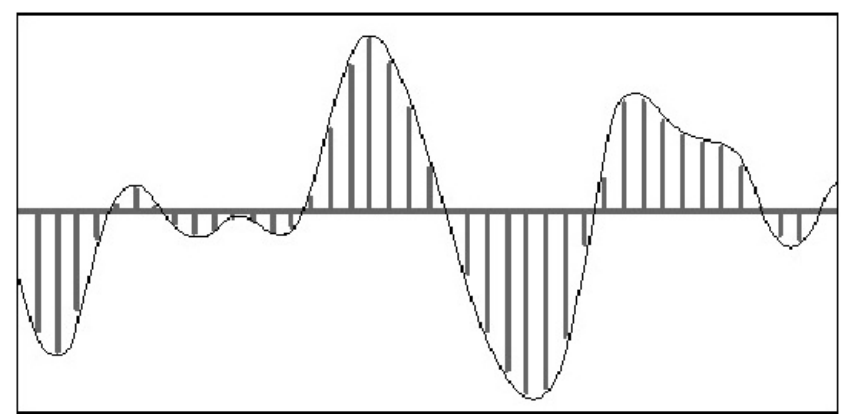

Figure 3. shows the representation of those voltages overlaid with the input AC sigma

Sample voltages overlaid with the input AC signal Now that the sound has been digitized, it can be stored via computer using any number of technologies, ranging from fast storage, such as main processor RAM, to off-line slow storage on magnetic tape. The actual speed of the storage medium is relatively unimportant with digital sound, since the bandwidth needed to accurately store the sound is relatively slow compared to most digital media [13]. Eventually, the sound needs to be played back. This is done via another electronic component that is the converse of the ADC: the digital-to-analog converter (DAC). The DAC is responsible for taking a digital value and converting it to a corresponding analog signal. To be effective, the conversion process needs to be the mirror image of that performed when converting the analog signal to digital. While the exact voltages Produced at the output of the DAC do not need to be 
identical to those seen at the input, they do need to be proportional to one another so that one waveform corresponds to the other. In addition, the samples need to be output at exactly the same rate that they were read in. Any deviation here will cause the output frequencies to be shifted up or down from the input, generally not a good thing [13].

Figure 4 shows the output of the DAC when given the same set of samples produced in Figure .2. At first glance, it seems that this is a radically different waveform. All the nice, smooth shapes shown in the earlier figures are gone, replaced by this stair-step, rectangular, artificial-looking creation.

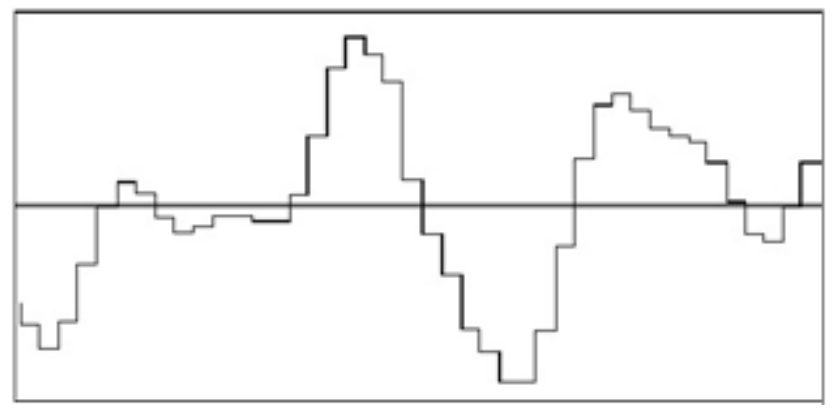

Figure 4. shows the output of the DAC when given the same set of samples produced in Figure .2.

DAC output Fortunately, Figure 4 is not that far removed from Figure 1. Mathematically, the sharp jumps that occur when we move from sample to sample represent high-frequency components in the output signal. These can (and must) be eliminated from the signal by means of a low-pass filter that lies between the output of the DAC and the final amplification stage of the audio output.

A low-pass filter is a network of electrical components designed to let frequencies below

a certain value pass through it unhindered, while attenuating frequencies above that point.

An ideal low-pass filter used with the samples shown here would completely stop any frequency above $4 \mathrm{KHz}$ and let frequencies below $4 \mathrm{KHz}$ pass through with no attenuation [14].

In practice, low-pass filters don't work perfectly, but even a low-budget filter can take Figure .4 and create a nearly indistinguishable copy of Figure 1. Without the filter, the sound sample will still be intelligible, but it will be filled with distracting highfrequency "noise" that is part of the reproduction process.

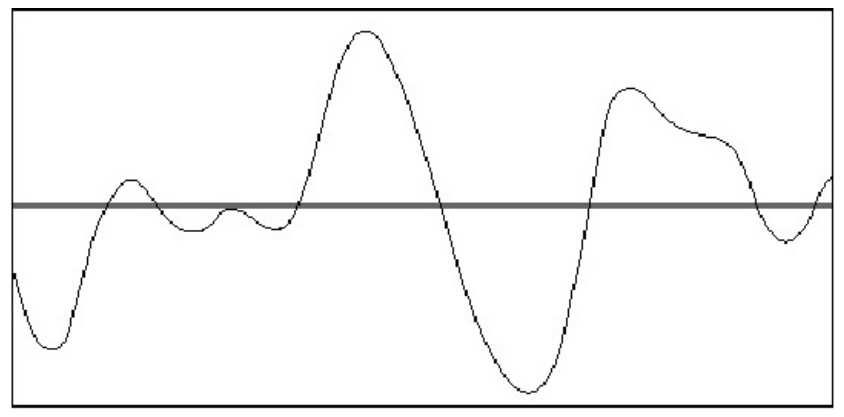

Figure 5, Sampling at a much higher rate.
Figure 5 shows the same figure when the sampling rate has been stepped up to a much higher rate. This increase in sampling rate clearly does a more accurate job of reproducing the signal. The next section discusses how variations in these parameters affect the output signal.

\subsection{Sampling Variables}

When an audio waveform is sampled, two important variables affect the quality of the reproduction: the sample rate and the sample resolution. Both are important factors, but they play different roles in determining the level of distortion produced when a sample is played back. The sample resolution is simply a measure of how accurately the digital sample can measure the voltage it is recording. When the input range is $-500 \mathrm{mv}$ to $+500 \mathrm{mv}$, for example, an eight-bit ADC can resolve the input signal down to about $4 \mathrm{mv}$. So an input signal of $2 \mathrm{mv}$ will either get rounded up to $4 \mathrm{mv}$ or down to $0 \mathrm{mv}$. This is called a quantization error [10]. Figure 6 shows the results of quantization error when sampling a waveform. In some cases the sample point has a larger magnitude than the audio signal, but in other places it has less. When the digitized signal is played back through a DAC, the output waveform will closely track the sample points, resulting in a certain amount of distortion.

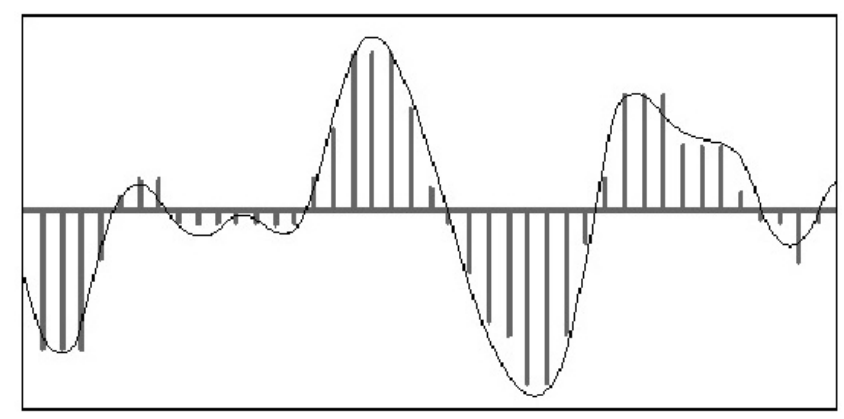

Figure 6, Quantization error when sampling a waveform

It might seem that eight bits should be enough to accurately record audio data, but this may not be the case because of the large dynamic range of audio the human ear can detect. If our $500 \mathrm{mv}$ range example were used, we might find that our input signal magnitudes range from $1 \mathrm{mv}$ to $500 \mathrm{mv}$ in a single recording session. The crash of drums in an orchestra could push the ADC to its limits, while a delicate violin solo may never go outside $5 \mathrm{mv}$. If the minimum digital resolution is only $5 \mathrm{mv}$, a very noticeable level ofDistortion will be introduced during this part of a recording session [15].

The sampling rate plays a different role in determining the quality of digital sound reproduction. One classic law in digital signal processing was published by Harry Nyquist in 1993. He determined that to accurately reproduce a signal of frequency $\mathrm{f}$, the sampling rate has to be greater than $2 * \mathrm{f}$. This is commonly called the Nyquist Rate. The audio signal in Figure 7 is being measured at a considerably slower rate than that shown in the previous examples, with noticeably 
negative consequences. At several places in the waveform it is not even sampled a single time during an excursion above or below the center line.

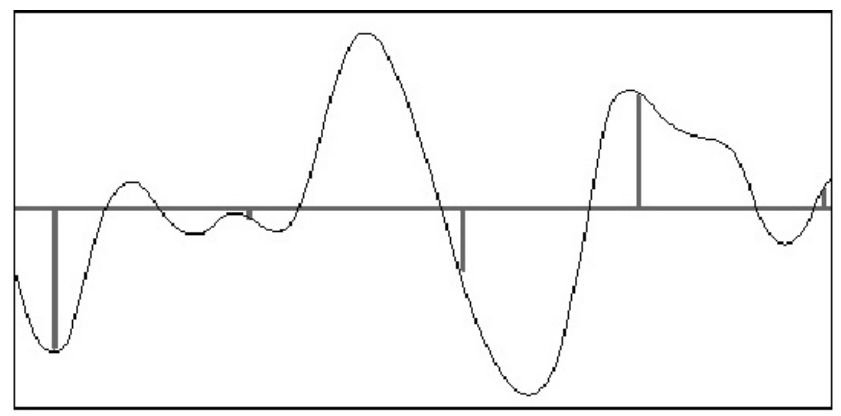

Figure 7. A slower sampling rate.

Figure 8 shows the waveform we could expect after playing back the digitized samples stored from Figure 7 . Clearly, after the digitized output is filtered, the resulting waveform differs quite a bit from that shown in the previous figure. What has happened is that the high-frequency components of the waveform have been lost by the slower sampling rate, letting only the low-frequency parts of the sample through.

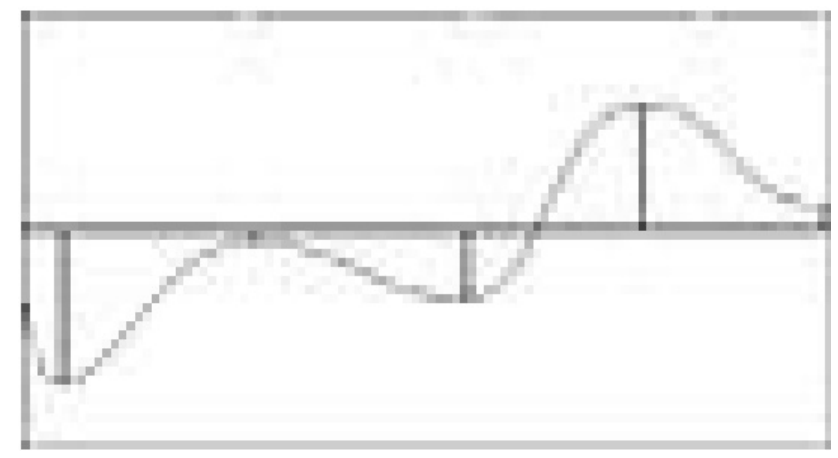

Figure 8. The waveform after playing back digitized samples.

The human ear hears sound up to $20 \mathrm{KHz}$, which implies that we need to sample audio to $40 \mathrm{KHz}$ or better to achieve good reproduction. In fact, the sampling rate used for digital reproduction of music via compact disk or digital audio tape is $44 \mathrm{KHz}$, using sixteen-bit samples. The quality of sound achieved at this sampling rate is generally acknowledged to be superior. This does not mean that all digital recordings have to be done at $44 \mathrm{KHz}$ rates [17]. Virtually every digital phone system in the world uses an $8 \mathrm{KHz}$ sampling rate to record human speech, with generally good results. This means that the phone system is unable to pass any signal with a frequency of $4 \mathrm{KHz}$ or higher. This clearly does not render the system useless - millions of long-distance calls over digital lines are made every day. The average speech signal is composed of many different frequencies, and even if everything above $4 \mathrm{KHz}$ is discarded, most of the speech energy still makes it through the system [18]. Our ears detect this loss as a lower-fidelity signal, but they still understand it quite well. The ultimate test of all this is how the audio output sounds to our ears. It is difficult to quantify a "quality of sound" measurement is strictly mathematical terms, so when discussing audio output, it is always best to temper judgments with true listener trials.

\section{Voice Message Silence Compression}

1. Determine threshold value that can be considered silence, even though it is not pure silence.

2. Extract the data from the sound file to be compressed pass through threshold check if it is below the threshold (considered to be silence) make it pure silence.

3. Run length coding to the manipulated data.

4. Store it as a compressed file.

\section{Flow Chart of Context Adaptive Algorithm of Silence Compression}
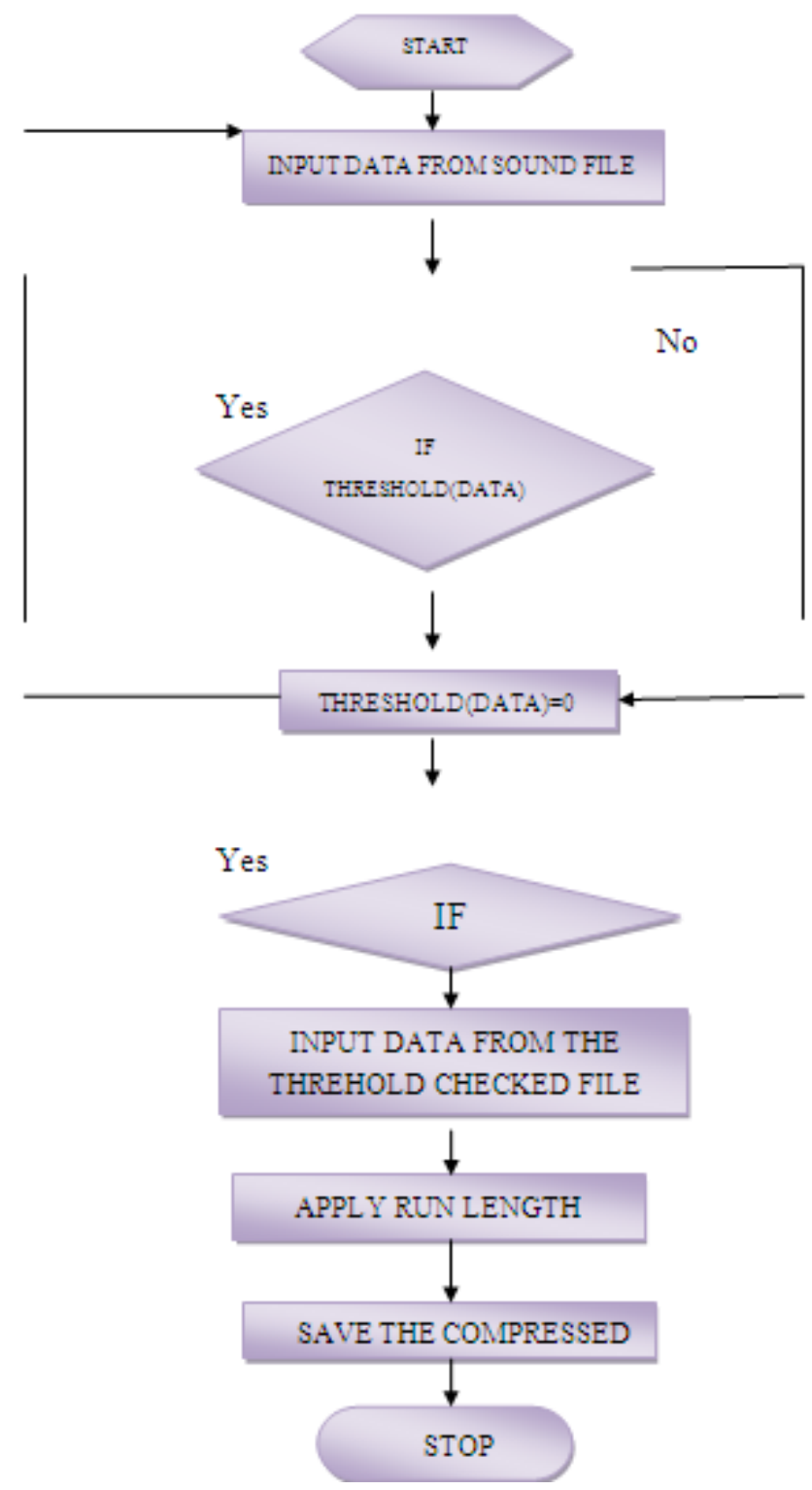
6.1. Experiments and Results

\begin{tabular}{|c|c|c|}
\hline File Name & Size in bytes & Bytes compressed \\
\hline 1)Original1.wav & 640440 & 1462 \\
\hline 2)Original2.wav & 499100 & 5000 \\
\hline 3)Original3.wav & 360100 & 4088 \\
\hline 4)Original4.wav & 986320 & 117781 \\
\hline 5)Original5.wav & 398600 & 45 \\
\hline
\end{tabular}

Sampled at $8000 \mathrm{hz}, 16$ bit

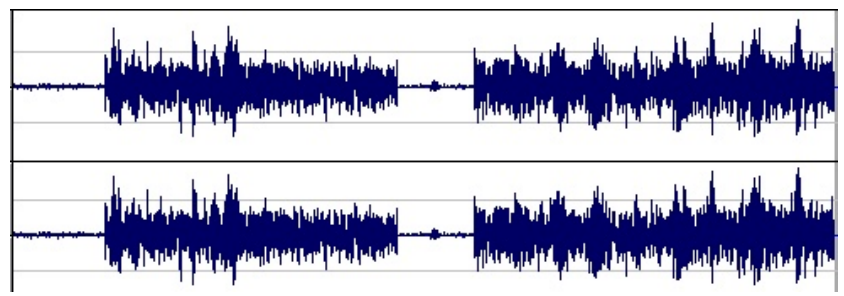

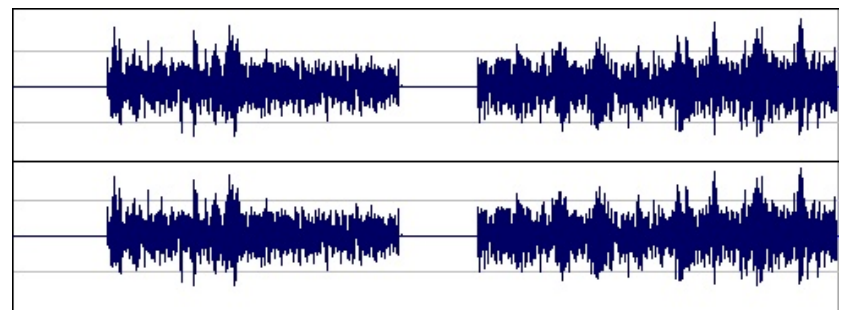

Original 2. wav after Silence compression /decompression

\subsection{Software Implementation Snapshots}

\section{2.1. Compression}

\begin{tabular}{|c|c|}
\hline$\Leftrightarrow$ SILENCE CO & $=\square x$ \\
\hline \multicolumn{2}{|c|}{ Enter File name to be compressed } \\
\hline COMPRESS & ABORT \\
\hline
\end{tabular}

\section{Original 2.wav}

\begin{tabular}{|l|l|}
\hline original2.wav & - \\
\hline COMPRESS & \\
\hline c.wav & \\
\hline
\end{tabular}

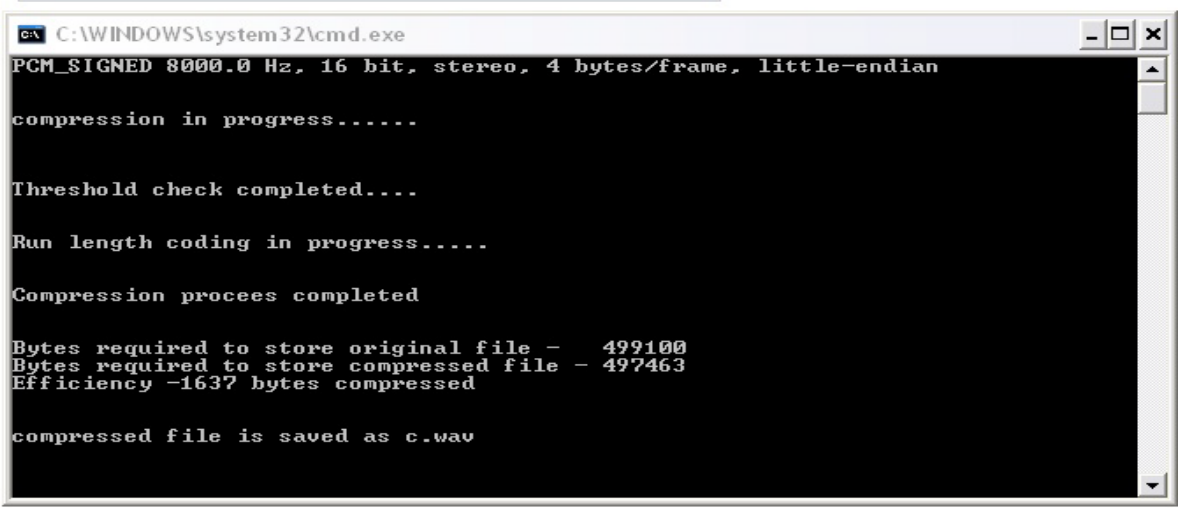

6.2.2. De-compression
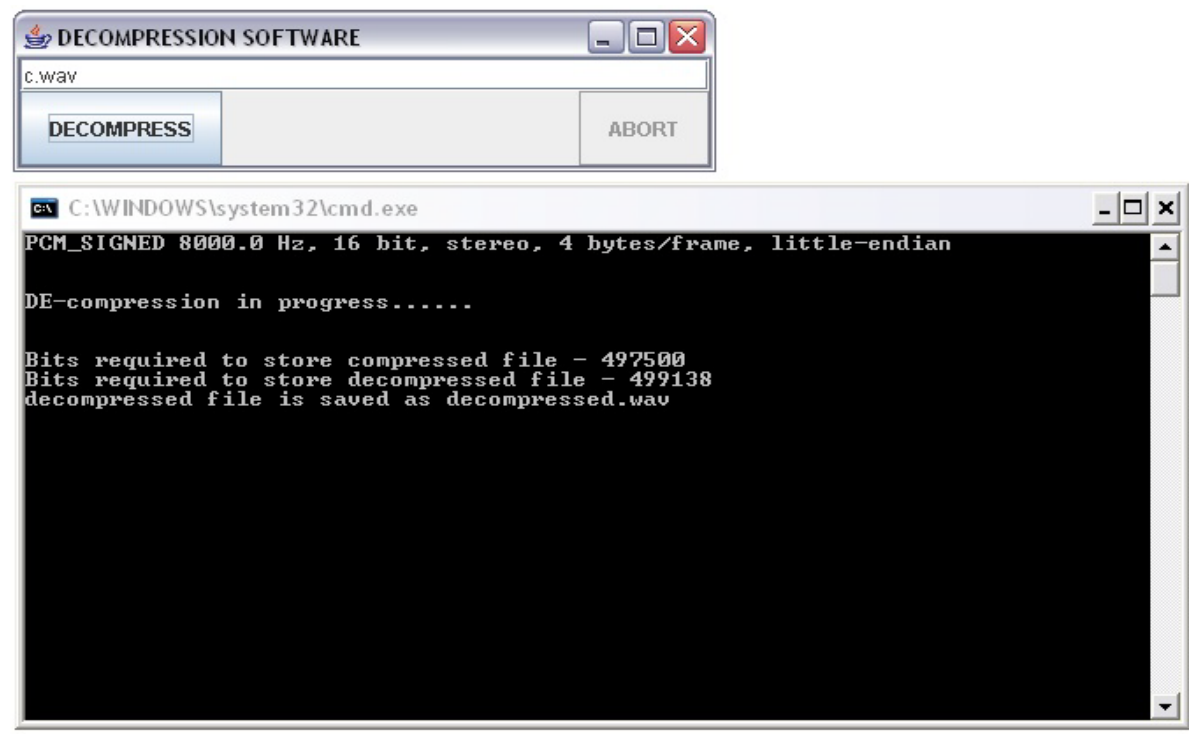


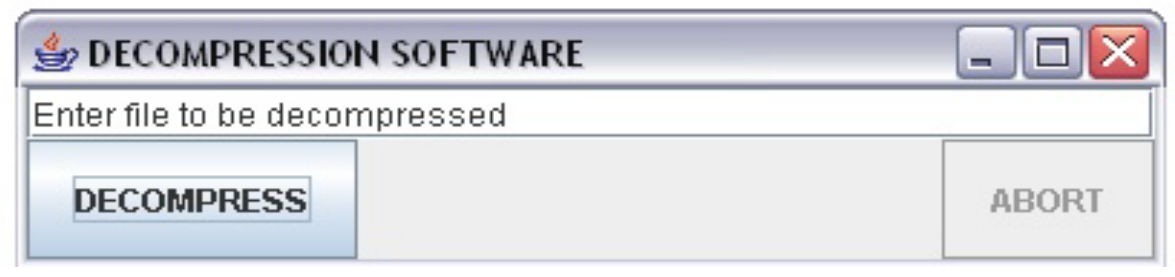

\section{Conclusions}

Voice message Silence compression system that improves data compression in a digital speech storage device, such as a digital telephone answering machine, without undue clipping of voice signals [19]. Instead of employing only real-time compression, the inventive silence system analyzes and compresses or re-compresses digital speech samples stored previously, when the voice messaging system is off-line or otherwise in a low priority state. A method of Context adaptive algorithm of Silence compression comprises receiving real-time speech samples, storing the same in memory, and analyzing the stored speech samples at a later time to determine thresholds for periods of silence [20]. The periods of silence are then compressed, and the silence compressed voice message is restored in memory. In this fashion, the processor is not required to make a silence period determination on-the-fly simultaneous with encoding and compression of the real-time voice message, and thus is not subjected to heavy processor loads typically encountered in real time. This enables more efficient compression of speech samples, lighter duty processors, and improved voice quality upon reproduction by eliminating undesired clipping of the voice signal encountered in prior systems after periods of silence. Voice message Silence compression speech samples is stored in a storage device for subsequent playback [21].

\section{REFERNCES}

[1] B.Xiao, Y.F.Huang, and S. Tang, "An approach to information hiding in low bit rate speech stream," in Proc. IEEE GLOBECOM 2008, Dec.2008, pp. 371-375, IEEE Press. Z.Wu, W.Yang, and Y.Yang, "ABS-based speech information hiding approach," Electron. Lett. vol. 39, no. 22, pp. 1617-1619, Oct. 2003

[2] Core java2 by Cay S.Horstmann and Gary Cornel,2008

[3] C.Krätzer, J.Dittmann, T. Vogel, and R. Hillert, "Design and evaluation of steganography for voice-overip," in Proc. IEEE Int.Symp. Circuits Syst., May 2006, pp. 2397-3234.

[4] Data compression book by Mark Nelson and Jean-Loup Gailly.,2008

[5] F. Huang, and C. Zhu, "Design and evaluation of steganography for voice- Steganalysis of compressed speech," in Proc. IMACS Multiconf. Computational Eng. Syst. Applicat.(CESA), Oct. 2006, pp. 5-10
[6] Hnyadav Spal, Design and development of voice authentication system-A brief Survey, varahmihir journal of computer \& information science, vol 3 no 122,pp, $85-96,2008$.

[7] hnyadav\&spal, "user identification by voice authentication system", pragyan journal of information technology,dec,2010 Ulrich's International Periodicals Directory, USA,.

[8] Hnyadav,Spal "Robust Mobile Phone User identification \& Voice Authentication",Technical Journal of LBSIMDS, vol-1 no 1 , july-dec-2010.

[9] Hnyadav, "Pattern Recognition with Artificial Neural Network.", pragyan journal of information technology, Volume 9 : Issue 1.pp 11 June 2011. Ulrich's International Periodicals Directory, USA

[10] N.Aoki,"A technique of lossless steganography for G.711 telephony speech," in Proc. 2008 4th Int. Conf. Intelligent Inf. Hiding Multimedia Signal Process. (IIH-MSP), Harbin, Aug. 2008, pp. 608-611.

[11] ] P.Chang and H. Yu, "Dither-like data hiding in multistage vector quantization of MELP and G.729 speech coding," in Proc. Conf. Rec. 36th Asilomar

[12] Sangwan, A., M.C. Chiranth, R. Shah, V. Gaurav and R.V. Prasad, Comparison voice activity detection algorithms for VOIP. IEEE Proc. Seventh Intl. Symp. Computers and Communications.2002.

[13] Li, X. and H.H. Yu, Transparent and robust audio data hiding in cepstrum domain. IEEE Intl. Conf. Multimedia and Expo, pp: 397-400.2000.

[14] L.Ma, Z. Wu, and W. Yang, "Approach to hide secret speech information in G.721 schemes" Lecture Notes Computer. Sci., vol. 4681, pp. 1315-1324, 2007.

[15] Markopoulou, A.P., F.A. Tobagi and M.J. Karam, Assessment of VOIP quality over internet backbones. Proc. Conf. Computer Communications (IEEE INFOCOM ), New York.

[16] Kida, Y. and T. Kawahara, 2004. Voice activity detection based on optimally weighted combination of multiple features. Kyoto 606-8501.2002.

[17] Michael Menth, Stefan Muehleck, Packet waiting time for multipltiplexed periodic on-off stream in presence of overlooking, International Journal of Communication Networking and distributed system vol. 4, Issue 2, pp 207-229, 2010.

[18] Ravichandran, T. and K. Duraiswamy, Implementation of quality of service to a voice over internet protocol networks. Intl. Conf. ICIS, Malaysia.2005.

[19] Ravichandran, T. and K. Duraiswamy, Performance 
Enhancement on Voice using VAD Algorithm and Cepstral Analysis, Journal of Computer Science 2 (11): 835-840, 2006.

[20] Srinivasan, K. and A. Gersho, Voice activity detection for cellular network. Proc. of the IEEE Speech Coding Workshop, pp: 85-86.,1993.

[21] Yong Feng Huang, Shanyu Tang," Steganography in Inactive Frames of VoIP Streams encoded by Source Codec" ieee transactions on information forensics and security, vol. 6 , no. 2, June 2011.

[22] Z.Wu and W.Yang, "G.711-based adaptive speech information hiding approach," Lecture Notes Comput. Sci., vol. 4113, pp. 1139-1144, 2006.

[23] Z.Wu, W.Gao, andW. Yang, "LPC parameters substitution for speech information hiding," J. China Univ. Posts Telecommun., vol. 16, no. 6, pp. 103-112, 2009

[24] http://www.cs.columbia.edu/ coms6181/slides/5/audio_long .pdf,2009

[25] http://www.jsresources.org/examples/audio_files.html,2010

[26] http://www.apl.jhu.edu/ hall/java,2010 